Recepción: 15 / 04 / 2017

Aceptación: 01 / 05 / 2017

Ciencias Educativas

Publicación: 15 / 05 / 2017

Revisión de Literatura

\title{
El papel de las Tics en el pensamiento crítico en educación superior
}

\author{
The role of Tics in critical thinking in higher Education
}

O papel das TIC no pensamento crítico no ensino superior

Lorenzo R. Fuentes-Campuzano ruffo.fuentesc@ug.edu.ec

Diva Y. Elizalde-Chiriboga" diva.elizaldec@ug.edu.ec

Juan E. Fernández-Escobar ${ }^{\text {m }}$ juan.fernandeze@ug.edu.ec

Correspondencia: ruffo.fuentesc@ug.edu.ec

Master en Nuevas Tecnologías Aplicadas a la Educación; Magister en Gerencia de la Educación Abierta; Economista; Universidad de Guayaquil, Ecuador.

II. Magister en Gerencia de la Educación Abierta; Economista; Universidad de Guayaquil, Ecuador.

III. Magister en Educación Superior; Licenciado en Ciencias de la Educación Especialización Informática; Profesor En Informática; Tecnólogo Pedagógico en Informática; Universidad de Guayaquil. Ecuador. 


\section{Resumen}

Dentro del marco de la sociedad actual, la educación superior debe asumir retos y compromisos para aportar desde la ciencia y la investigación a las necesidades de cambio a favor del bienestar común, la ética y la democracia, donde el trabajo no sea una mercancía más del sistema que se compra y vende en función del lucro, dejando de lado los derechos, la familia, la libertad y respecto a la dignidad del ser humano. Este artículo tiene como propósito establecer el rol de las Tecnologías de la Comunicación e Información dentro del paradigma de la Pedagogía Crítica para desarrollar un pensamiento en la educación superior que aporte a esos cambios. El análisis se lo ha realizado en base a una metodología de examen bibliográfico, observaciones de conductas de actores del entorno socioeducativo y fundamentalmente en exploración de procesos hermenéuticos.

Palabras clave: Tecnologías de la comunicación e información; pensamiento crítico; modelo pedagógico; globalización. 


\begin{abstract}
Within the framework of today's society, higher education must assume challenges and commitments for science and research in the needs of change a favor of common welfare, ethics and democracy, where work there is no sea and merchandise more System Which is bought and sold according to profit, leaving aside the rights, family, freedom and respect for the dignity of the human being. This article aims to establish the role of Communication and Information Technologies within the paradigm of Critical Pedagogy to develop a thinking in higher education that the sport to the changes. The analysis has been made on the basis of a methodology of bibliographic examination, observations of the behavior of the actors in the socio-educational environment and fundamentally in the exploration of hermeneutic processes.
\end{abstract}

Key words: Communication and information technologies; critical thinking; pedagogical model; globalization. 


\section{Resumo}

No âmbito da sociedade de hoje, o ensino superior deve assumir desafios e compromissos para a ciência e pesquisa nas necessidades de mudança um favor de bem-estar comum, ética e democracia, onde o trabalho não há mar e mercadorias mais Sistema Que é comprado e vendido de acordo com Deixando de lado os direitos, a família, a liberdade eo respeito pela dignidade do ser humano. Este artigo pretende estabelecer o papel da Comunicação e Tecnologias de Informação dentro do paradigma da Pedagogia Crítica para desenvolver um pensamento no ensino superior que o esporte às mudanças. A análise foi feita com base em uma metodologia de exame bibliográfico, observações do comportamento dos atores no ambiente sócio-educacional e fundamentalmente na exploração de processos hermenêuticos.

Palavras chave: Tecnologias de comunicação e informação; pensamento crítico; modelo pedagógico; globalização.. 


\section{Introducción.}

Las Tecnologías de la Información y la Comunicación han impulsado extraordinariamente el fenómeno conocido como globalización. Las Tics como avance tecnológico han permitido la implantación de la sociedad del conocimiento. La Internet como uno de los resultados más importantes del desarrollo tecnológico disminuye los tiempos, distancia y costos. Por medio de la Internet las culturas de las distintas comunidades del mundo trascienden sus territorios y se hacen conocidas por otras sociedades. El arte tomado de forma individual ha alcanzado un alto nivel de socialización a través de la Internet. Las transacciones económicas de todo tipo son mucho más eficaces. El conocimiento científico ha tenido lo suyo, gracias a la red, la ciencia y la investigación se han desarrollado grandemente debido a la producción de material que ha sido socializado y servido de marco epistemológico para la producción de nuevo conocimiento. Dentro de este contexto, la apertura de los mercados de la mayoría de los países del mundo ha facilitado la inversión externa, como implantación del modelo neoliberal de la economía: transnacionalización de los grandes capitales, condicionamiento de los organizamos multilaterales de crédito a países pobres. En fin lo anterior es una descripción acertada de los que conocemos como globalización. Cabe preguntarse ¿Se encuentran las Tecnologías de la Comunicación e Información enteramente al servicio del neoliberalismo bloqueando el desarrollo del pensamiento crítico? ¿En qué medida las Tecnologías de la Comunicación e Información sirven para general pensamiento crítico en la educación superior? Estas serán entre otras las interrogantes a las que daremos respuesta en el presente análisis. 


\section{Las Tics en el proceso de globalización}

Octavio Ianni en su libro “Teorías de la Globalización” cita los diferentes nombres dados a la presente época por distintos autores; así tenemos: Primera revolución mundial (Alexander King), Tercera Ola (Alvin Tofler), Sociedad Informática (Adam Schaff), Sociedad Amébica ( Kenicho Ohmae), Aldea Global (McLuhan) entre otros. Y específicamente a la Globalización se la conoce como "Economía del mundo", Sistema-mundo", "Shopping center global", "Disneylandia global", "Nueva división internacional de trabajo" entre otros denominativos.

El término Aldea Global hace alusión a la era cibernética en términos de que las diferentes culturas y civilizaciones estarán articuladas y trascenderán sus fronteras gracias a la tecnología (Ianni 2002). En este mismo marco se describe todo lo que a través de las Tics se puede alcanzar para ser nuestro mundo más fácil. Al fin y al cabo para qué es la tecnología sino para resumir y obviar proceso y hacer el trabajo más eficiente. Cabría preguntarse qué pasaría a largo plazo con el concepto de identidad cultural de las sociedades. ¿Serán acaso las Tics un medio de alienación cultural? Eso dependerá de las políticas de cada país para el tratamiento y uso conveniente de estas herramientas y del fomento de los valores nacionales y de identidad cultural de su sistema educativo.

La Tics en tanto que mass media forman parte protagónica dentro del proceso de globalización capitalista. Siendo los medios de comunicación grandes emporios, dan información maquillada y censuran la realidad con el fin de justificar las políticas neoliberales y el papel de los regímenes hegemónicos globales en la aniquilación de personas y gobiernos que se oponen a sus intereses e ideologías para la obtención de recursos y demostración de fuerza. 
Agrega Ianni:

Los medios de comunicación entonces, son sensibles a las reivindicaciones de diversos grupos y clases sociales. De esta forma los medios de comunicación expresan lo que sucede en el mundo. Simultáneamente estos medios operan en consonancia con los centros de poder mundiales. Los acontecimientos son presentados como un espectáculo desterritorializado y ahistórico. (Ianni 2002)

Ignacio Ramonet creó un concepto llamado "El pensamiento único" que no es otra cosa que la ideología totalitaria capitalista como única opción de pensamiento en el mundo que surgió a partir de 1989 después del derrumbe del muro de Berlín. Esta ideología tiene que ser llevada a los habitantes del mundo a través de los medios de comunicación.

Rubido, Aparici, Díez y Tucho (s.f) manifietan “Toda esta teoría económica del pensamiento único se presenta a sí misma corno algo "natural" e irremediable: gusten o no, nos dicen que las cosas son así y no pueden ser de otra manera". El objetivo es claro, el público es llevado a una situación de desaliento y desesperanza. La sociedad mayoritariamente no preparada para asimilar críticamente la realidad social es presa del engaño y vilmente manipulada por los medios.

Venezuela, un país extremadamente rico en recursos naturales, donde una crisis económica es lo último que se esperaría, se encuentra actualmente en grave crisis de orden político ideológico, producto de un sabotaje económico en donde la prensa cotidianamente oculta la realidad, y presenta los hechos malamente contrastados. 
La revista Íconos de Flacso presentó un artículo Titulado "De mediadores a protagonistas. Crisis política, medios y comunicación en Venezuela” donde expresa

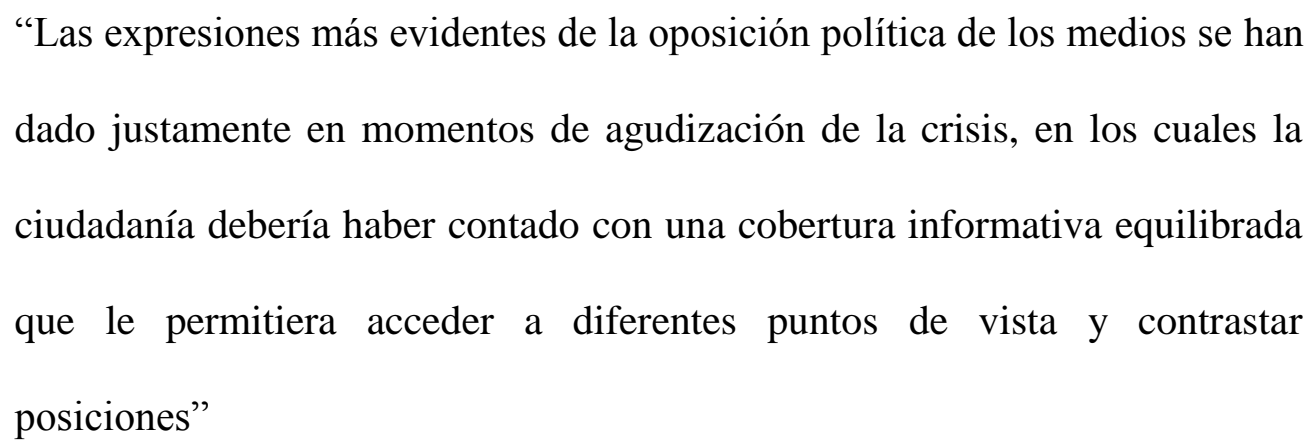

El papel de los medios de información es explícitamente el de mantener el statu quo dentro del sistema capitalista y convencer a las personas que solo existe un pensamiento único y una sola ideología para lo cual engaña, oculta, y manipula la información.

Otras formas de manipulación y alienación de los medios es vender la idea, el falso valor, conocido como sueño americano. Se entiende por sueño americano un conjunto de valores y conductas preconcebidas dentro del sistema capitalista basados en el egoísmo del tener y no del ser, del placer y no del sentir, de consumir y no de vivir. Un sistema donde las sociedades no son tal sino mercados y los individuos no son sino consumidores. Una sociedad asentada en el individualismo donde el objetivo de vida es llegar a ser rico, situación que muy pocos alcanza debido a las estructuras del sistema económico basadas en la explotación, siendo los ciudadanos víctimas de su propia ideología.

Las programaciones de los medios televisivos y cinematográficos son el vector contaminante en este proceso de enajenación ideológica en este caso. Noan Chomsky manifiesta al respecto: 
"La mayoría de los individuos tendría que sentarse frente al televisor y masticar religiosamente el mensaje, ese que dice que lo único que tiene valor en la vida es poder consumir cada vez más y mejor, y vivir igual que esa familia de clase media que aparece en la pantalla y exhibe valores como la armonía y el orgullo americano. Puede que usted piense que ha de haber algo más, pero en el momento en que se da cuenta de que está sólo, viendo la televisión, da por sentado que ésto es todo lo que existe ahí fuera, y que es una locura pensar en que existe otra cosa" (Chornsky: 1995).

Nos encontramos ante una situación preocupante cuya solución debe estar en el ámbito de la educación. Pero sabemos por otro lado que la educación es parte de la superestructura social y será siempre condescendiente con las estructuras capitalistas preconcebidas. ¿Qué hacer?

McLaren (1997) manifiesta:

"En el debate educativo de nuestros días no se habla de la desesperada necesidad que tienen nuestras escuelas de crear una ciudadanía alfabetizada en el lenguaje de los mass media, capaz de interrumpir, contestar y transformar el aparato de estos, de manera que pierdan su poder de infantilizar a la población y dejen de crear sujetos sociales pasivos temerosos, paranoicos y apolíticos" (p. 26)

Y afirma:

Los educadores se han de percatar de que es totalmente imposible alcanzar un nuevo orden mundial sin crear antes en casa un nuevo orden moral (es 
decir en las aulas y en los hogares de la nación) -un orden moral que rechace el reto a las verdades recibidas y a las convicciones acertadas que ha provocado la actual crisis de historia e identidad-. Hasta Ahora Busch y Clinton han tenido éxito al crear un nuevo orden moral en el que la gente joven es capaz de resistir la tentación de entrar en cualquier lógica de oposición a través de las esferas públicas de la resistencia cultural...” (p. 27).

Desde la fecha en que McLaren expresa los comentarios citados ocurrió un hecho importante de expresión de masas luego de la crisis de la burbuja inmobiliaria en el año 2008 que fue la toma de los espacios del Zuccotti Park de Lower Manhattan en la Ciudad de Nueva York, Estados Unidos y la estructuración del movimiento Occupy Wall Street. Es importante considerar que el movimiento no se ha vuelto a manifestar, probablemente por la ausencia de líderes, ya que la manifestación originalmente estuvo a cabo de la revista Adbusters. En consecuencia podemos manifestar que las palabras de McLaren tienen vigencia.

\section{La Tics y el modelo pedagógico}

El desarrollo del pensamiento crítico está basado en el modelo pedagógico. Las Tics en este contexto cumplen funciones más específicas.

La Naciones Unidas citadas por Fëdorov (s.f) manifiestan:

El informe de la Organización de las Naciones Unidas para la Educación, la Ciencia y la Cultura (UNESCO) generado por el equipo de Jacques Delors (1996), plantea el reto particular que enfrenta la educación superior de 
potenciar la comprensión, despertar la curiosidad intelectual, estimular el sentido crítico y adquirir al mismo tiempo autonomía de juicio. La Declaración sobre la educación superior en el siglo XXI, elaborada por la conferencia mundial de la UNESCO, que fue organizada en 1998 en París, refuerza dicha posición y expone que: "Las instituciones de educación superior deben formar a los estudiantes para que se conviertan en ciudadanos bien informados y profundamente motivados, provistos de un sentido crítico y capaces de analizar los problemas de la sociedad, buscar soluciones, aplicarlas y asumir responsabilidades sociales".

Después de leer esta declaración vale indicar que tras la realización de observaciones perpetradas a los estudiantes universitarios se puede ver claramente la falta de conocimiento de la realidad mundial de los estudiantes superiores de la universidad de Guayaquil. En los contenidos académicos y en los discursos de los docentes universitarios no se escuchan términos como igualdad, equidad, clases sociales, o lucha de clases pero se oye mucho hablar sobre pensamiento crítico.

Según Paulo Freire un sujeto con pensamiento crítico tiene las siguientes características:

- Independiente, se construye a partir de la individualidad.

- Informado, requiere conocimientos e información.

- Cuestionador, plantea preguntas o problemas que le interesan y que busca resolver.

- Razonado, que a través de la capacidad adquirida de ciencia intenta argumentar.

- Social-socializado, que compara, contrasta y comparte su raciocinio (Klooster, 2001, en Cassany, 2006b: 70). 
Según Paulo Freire, estas características del pensamiento crítico, implican que el hombre comprenda su posición dentro de su contexto socio-cultural e histórico. Esta concienciación debe darse a partir del trabajo pedagógico crítico, fomentado por el diálogo equitativo entre profesor y alumno.

Roberto Aparici (2001), citado por Fëdorov (s.f) Indica: “La incorporación de redes, no significa, en líneas generales, una propuesta pedagógica y metodológica distinta si, previamente, no se las ha integrado y desarrollado en función de un modelo comunicativo y pedagógico distinto”.

Y concluye:

Sin embargo, ya se vislumbra un panorama educativo diferente, donde a las TIC, debido a su capacidad de traspasar las barreras espacio-temporales, se les otorga un rol mayor, tal vez decisivo y de dimensiones que aún no se conocen en toda su amplitud y profundidad. Ahora nos damos cuenta de que en el transcurso de una década, los pronósticos de Pérez (1994) se han cumplido y las tecnologías de la información y la comunicación han potenciado el bagaje metodológico de la docencia comprometida con el desarrollo del pensamiento crítico de los estudiantes, en el contexto de la pedagogía crítica y del conocimiento.

Es decir que queda entendido que el pensamiento crítico en términos de proceso educativo no viene dado por las Tecnologías en sí mismo sino por el modelo pedagógico. Más adelante abordaremos el papel de la Tics en este contexto. 
Mientras tanto de acuerdo a lo manifestado por Fodorov haremos un breve análisis del concepto y caracterización de un modelo pedagógico.

Para poder entender mejor el concepto de un modelo pedagógico analicemos primeramente qué es un MODELO para luego caracterizar la PEDAGOGÍA en sus connotaciones de ciencia y proyecto social.

De acuerdo a Flores Ochoa (1994):

"Un modelo es la imagen o representación del conjunto de relaciones que definen un fenómeno, con miras a su mejor entendimiento. De acuerdo con esta definición puede inferirse que un modelo es una aproximación teórica útil en la descripción y comprensión de aspectos interrelacionados de un fenómeno en particular. En esta conceptualización de modelo es necesario establecer que el análisis del fenómeno en estudio no es únicamente un proceso analítico en el cual el todo es examinado en sus partes, sino también como un proceso de integración de relaciones”. (p. 60)

Flores Ochoa claramente aborda el problema desde el punto de vista sistémico. Es decir análisis y síntesis para estudiar la parte y el todo, dentro del sistema donde cada uno de sus componentes, con sus funciones propias, está relacionado y se afectan directa o indirectamente.

Una de las características importantes de un modelo es su connotación teórica que interpreta una realidad abstracta a nuestra percepción y que intenta descubrirla para una mejor comprensión de la realidad concreta. 
En cuanto al concepto de pedagogía de acuerdo a la Dra. Daysi Hevia Bernal "Es un conjunto de saberes que buscan tener impacto en el proceso educativo, en cualquiera de las dimensiones que este tenga, así como en la comprensión y organización de la cultura y la construcción del sujeto".

Constituye la pedagogía así un conjunto de conocimientos específicos fundamentados en teorías del aprendizaje, sicológicas, sociológicas y metodológicas.

Por otro lado la pedagogía según Zapatier es un Proyecto Social porque forma parte esencial en el proceso de socialización del ser humano dentro de un centro educativo especialmente destinado para cumplir con unos fines específicos, de manera intencionada deliberadamente establecida, por ello se dice que la pedagogía es parte del proceso de socialización de segundo piso. Llamándose de segundo piso o secundaria a aquella que experimenta el niño fuera de su núcleo familiar, en su contacto directo con lo público, mientras que la primaria corresponde al conjunto de valores y costumbres que se dan dentro del seno de la familia.

La pedagogía como proyecto social se entiende como una política de estado en educación, y en todas aquellas variables socioeconómicas, políticas, jurídicas y culturales que están relacionadas con ella en una suerte de contextualización dinámica.

En cuanto al Modelo Pedagógico podemos establecer las siguientes características: 
1. El modelo pedagógico sistematiza las interrogantes y componentes curriculares:

\begin{tabular}{|c|c|}
\hline Interrogantes curriculares & Componentes curriculares \\
\hline ¿Para qué enseñar? & Objetivos \\
\hline ¿Qué enseñar? & Contenidos \\
\hline ¿Cuándo enseñar? & Secuencia, edad \\
\hline ¿Cómo enseñar? & Metodología \\
\hline ¿Con qué enseñar? & Recursos, medios \\
\hline ¿Qué y Cómo se evalúa? & Evaluación. \\
\hline
\end{tabular}

Un modelo pedagógico queda definido de acuerdo a la respuesta que dé a cada una de las interrogantes pedagógicas planteadas.

2. Una de las características fundamentales de un modelo pedagógico es la primera pregunta, que constituye a su vez el norte del proceso pedagógico, la finalidad principal del Proyecto Social. ¿Para qué enseñar?

3. El modelo pedagógico salta a la vista tanto en la teoría como en la práctica docente. Es decir que los principios metodológicos, objetivos, etc. que utilice el maestro no pueden definir por sí solos un modelo pedagógico si no van acompañados de la correspondiente práctica docente: la relación alumno-maestro, los recursos, etc.

4. Todo modelo pedagógico está basado en principios sicológicos. Se refiere básicamente a que todo modelo pedagógico debe tomar al ser como una trilogía: Cognitivo, expresivo y afectivo o denominados de manera diferentes pensar, actuar y sentir o cognitivo, procedimental y actitudinal. Es por esto que todo aprendizaje debe estar dispuesto a desarrollar estas tres áreas de la sicología.

5. El modelo pedagógico representa un enfoque de un conjunto de teorías en el ámbito de sicología, sociología, filosofía (epistemología, ontología, axiología, metodología) y 
pedagogía. Por este motivo es que se clasifica al modelo pedagógico como una Teoría Educativa que a su vez está conformada por otras teorías a nivel interdisciplinario.

6. Todo modelo pedagógico procura efectividad del proceso de enseñanza aprendizaje.

7. Todo modelo pedagógico tiene un fundamento ideológico implícito. Es precisamente en esta característica de modelo pedagógico donde se decide cuál es el tipo de individuo que se desea formar, cuál es la respuesta a la primera interrogante del currículo, cuáles son los contenidos científicos que van a desarrollarse y cuál es el Proyecto Social al que se debe el modelo pedagógico elegido. Decimos esto porque a cada orden social establecido corresponden unos objetivos educativos para formar ciudadanos que vayan a la reproducción del sistema u orden social imperante y lo reproduzca. Así en un orden capitalista se necesita formar individuos emprendedores o subalternos obedientes dependiendo en la clase social en que se eduquen.

Es importante destacar que a cada sistema económico le corresponde un modelo pedagógico. Tenía razón Aparici citado anteriormente cuan decía que las tics no tendrían un cambio sustantivo al proceso educativo si no se revisa el Modelo Pedagógico (Aparici 2001).

Por otro lado en la definición de Modelo pedagógico observamos que la Tics forman parte de los procesos metodológico y de los recursos a utilizar dentro del modelo. Cuando nos planteamos las interrogantes de Cómo enseñar y Con qué enseñar la tecnologías responden en parte a lo primero y en gran medida a lo segundo.

Pere Marqués (2012) realiza una clasificación del uso de las Tics, así tenemos: como medio de expresión y para la creación, canal de comunicación, instrumento para procesar información, 
fuentes de información, organización y gestión de los centros, tutoría, recurso interactivo para el aprendizaje e instrumento cognitivo.

Esta clasificación del uso o de las funciones de las Tics es crucial para entender su papel en el desarrollo del pensamiento crítico. En vista de que las Tics forman solo parte de un rubro (digámoslo así) del modelo pedagógico, y además que el pensamiento crítico es en esencia dos cosas, por un lado, el desarrollo de unas capacidades cognitivas resumidas en análisis, síntesis, evaluación y proposición, y por otro, la adopción de una epistemología dialéctica histórico lógica de la realidad; podemos afirmar sin temor a equivocarnos que no existe una correlación entre las nuevas tecnologías y el desarrollo del pensamiento crítico si no hay una reorientación en los procesos metodológicos y de modelo pedagógicos.

\section{El pensamiento crítico}

El concepto de pensamiento crítico está en función del modelo pedagógico. Muchos docentes hablan sobre pensamiento crítico, pero este puede tener acepciones diferentes. Es necesario tener en cuenta cuántas acepciones diferentes de pensamientos críticos existen de acuerdo a diferentes ópticas. Así vemos definiciones que tienen que ver con conocimiento de su realidad objetiva: "Capacidad que tiene el ser humano para construir una representación e interpretación mental significativa de su relación con el mundo". (Villarini s.f). En el común de los docentes de educación superior el pensamiento crítico obedece a una situación de adaptabilidad.

En un contexto más importante tenemos el pensamiento crítico no adaptativo sino transformador. Así tenemos el marxismo como epistemología de pensamiento crítico. Morales Zúñiga manifiesta: 
...el marxismo es pensamiento crítico, pues además de evaluar las condiciones de validez de la razón económica de su época, se convierte, también, en crítica social, en la medida en que denuncia y cuestiona realidades como la desigualdad social, la explotación de una clase sobre otra, la enajenación y alienación, así como el dominio ideológico que no es sino otra manifestación de la dominación de clase.

Y añade: "En esta operación del marxismo vemos puesta en práctica la citada tesis once sobre Feuerbach, donde Marx establecía como función de la Filosofía no solo la comprensión del mundo, sino su transformación” (Morales Zúñiga 2014).

En un mundo globalizado dominado por el capital comercial y el neoliberalismo al pensamiento crítico hay que darle una significación de cambio. Las estructuras actuales de explotación deben cambiar. El estudiante universitario y la educación superior deben ser direccionados en términos del pensamiento crítico transformador y no adaptativo.

\section{Conclusión.}

En virtud de lo analizado las Tics pueden ayudar en cualquier proceso cognitivo, es decir con ellas nos podemos hacer más revolucionarios o más alienados, más analíticos o más distraídos. El uso que cada individuo o cada sociedad o cada sistema educativo le dé a las tecnologías está en función de la voluntad de cada individuo, cada sociedad o cada sistema. Las Tics no son buenas o malas per se. Es necesario una política deliberada que contemple objetivos hacia dónde habrá que enrumbar a la educación en términos de proyecto social. 
El pensamiento crítico se desarrollará con la implantación de un modelo pedagógico cuyos objetivos estén en función de una transformación positiva de la sociedad hacia el buen vivir. El constructivismo como modelo pedagógico propone un pensamiento crítico adaptativo. Los docentes de los centros educativos por ser formados dentro de ese modelo reproducen el modelo.

Los contenidos para el desarrollo de pensamiento crítico están en función de dos componentes: por una parte el desarrollo de habilidades del pensamiento y por otra un método dialéctico histórico lógico que logre capacidades holísticas y sintéticas, que analice la realidad desde el origen de su proceso histórico.

\section{Bibliografía.}

Andrei, N., \& Fedorov, F. (2012). Siglo XXI, la universidad, el pensamiento crítico y el foro virtual. Revista Iberoamericana de Educación, 1-10.

Bernal, D. (2010). Arte y pedagogía. México: Portal de la Red de Salud de Cuba.

Cerbino, M. (2003). Medios, politica y democracia. ÍCONOS, 16(1), 24-29.

Flórez Ochoa, R. (1994). Hacia una pedagogía del conocimiento Santafé de Bogotá. México: McGraw-Hill.

Ianni, O. (1996). Teorías de la globalización. México: Siglo XXI editores CEIICH-UNAM.

Morales Zúñiga, L. (2014). El pensamiento crítico en la teoría educativa contemporánea. Revista Electrónica "Actualidades Investigativas en Educación", 14(2), 1-23.

Pere Marqués, G. (2010). Impacto de las tic en la educación: funciones y limitaciones. Revista de investigación, 1-15.

Villarini Jusino, Á. (2014). Teoría y pedagogía del pensamiento crítico. PERSPECTIVAS PSICOLÓGICAS, $3(4), 35-42$. 